\title{
An image encryption scheme employing key related skipping
}

\author{
Jakub Oravec, Ján Turán, L’uboš Ovseník, Tomáš Huszaník ${ }^{*}$
}

\begin{abstract}
This paper describes an image encryption algorithm which utilizes chaotic logistic map. Values generated by this map are used in two steps of algorithm which shuffles image pixels and then changes their intensities. Design of the encryption scheme considers possibility of various attacks, such as statistical, differential or phase space reconstruction attacks. Robustness against last mentioned type of attacks is introduced by selective skipping of values generated by the map. This skipping depends on key entered by user. The paper also verifies properties of proposed algorithm by common measures and by set of statistical tests that examine randomness of computed encrypted images. Results are compared with other approaches and they are also briefly discussed.
\end{abstract}

K e y w or d s: image encryption, iterate skipping, logistic map, phase space reconstruction attack

\section{Introduction}

Security of various kinds of data has become an interesting topic in last years. Majority of applications could use already proposed solutions, however there are still some specific tasks which require unique approaches. One example of these is the field of image encryption, where properties of images such as large amount of pixels and high values of inter-pixel correlation are taken into account.

These properties and also the design of encryption algorithms could cause insufficient encryption results. For example, let us illustrate the performance of Advanced Encryption Standard (AES) for purposes of image encryption. In its simplest mode of operation (Electronic CodeBook, ECB [1]), the plaintext image which is going to be encrypted is divided into blocks with size of 128 bits (16 grayscale pixels). If plaintext image contains blocks with identical pixel intensities on various locations, the encryption produces a set of blocks with corresponding encrypted intensities on these locations. A demonstration of this property is shown in Fig. 1.

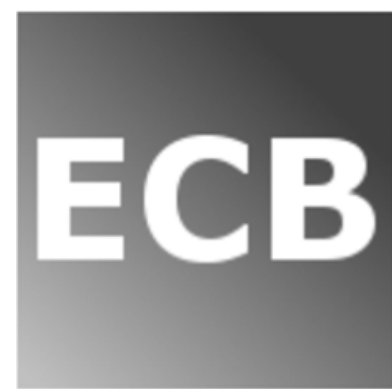

plaintext image

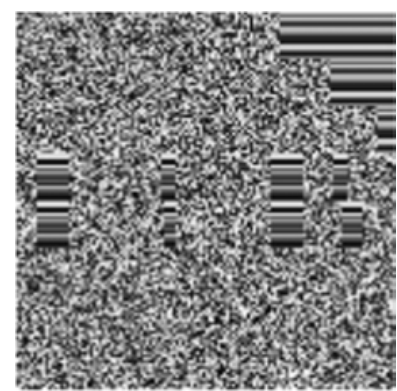

encrypted image
Fig. 1. Illustration of block effect occurring in ECB mode of AES
It could be stated that dark region with small intensity changes in upper right corner of image produces identical blocks in the same corner of encrypted image. Blocks inside white characters, which do not contain background pixels also create visible artefacts. Similar weaknesses could increase possibility of a successful statistical attack.

The dependencies between intensities of image pixels could be introduced by ciphertext chaining. This principle is used in another mode of AES, CipherBlock Chaining (CBC) [1]. The first block uses so called initialization vector which modifies pixel intensities by means of eXclusive OR (XOR). Following blocks use encrypted pixel intensities instead of initialization vector. This approach greatly suppresses possibility of statistical attacks, however, some algorithms could reach similar results with smaller computational complexity.

One of alternative solutions for purposes of image encryption is usage of chaotic encryption algorithms. First proposal of these techniques was described in late 1980s by Matthews [2]. Since then, many approaches were presented including one from Fridrich [3], where the encryption algorithm was divided to two stages. In the first stage, called confusion, the image pixels change their locations in order to minimize correlation between adjacent pixels. After that, diffusion takes place. During this stage, the dependencies between intensities of image pixels are created. Also the values computed by iterating the chaotic map are used for modifying the resulting intensities of pixels in encrypted image.

However, chaotic encryption algorithms have also some drawbacks. First of all, some approaches use two dimensional chaotic maps for pixel shuffling during confusion stage. As majority of these maps was originally proposed for usage on unit square $[4,5]$, their corresponding discrete versions work only with images which have square

Department of Electronics and Multimedia Communications, Faculty of Electrical Engineering and Informatics, Technical University of Košice, Košice, Slovakia, jakub.oravec@tuke.sk, jan.turan@tuke.sk, lubos.ovsenik@tuke.sk, tomas.huszanik@tuke.sk 


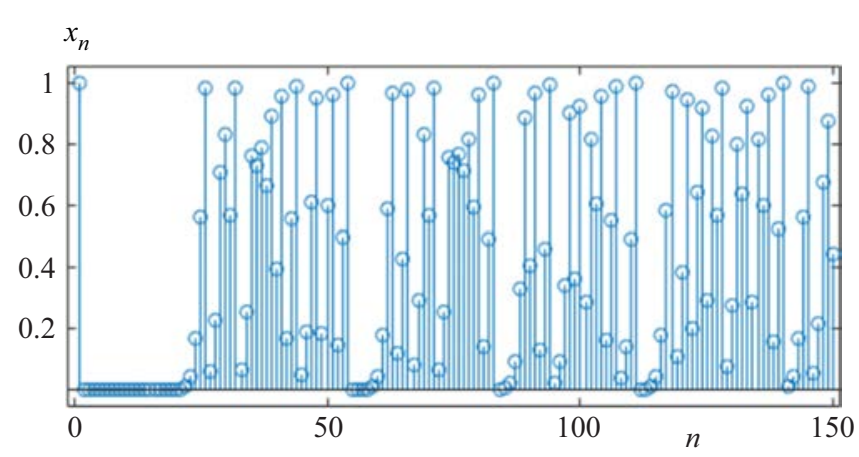

Fig. 2. Transient period occurring during first iterations of logistic map

resolution ( $n \times n$ pixels). Also, some maps have so called fixed points, which are locations that do not change their coordinates in following iterations of the map.

Usage of chaotic maps during diffusion could also cause problems when they are employed for generating pseudorandom sequence (PRS) which modifies pixel intensities. In this paper, we would like to point out these problems, describe their importance and propose a solution, which deals with troubles caused by not suitable usage of chaotic maps.

\section{Related work}

As it was already mentioned, chaotic maps could be used during diffusion stage of image encryption algorithms. One example of these maps is logistic map (LM), which was popularized mainly by May [6]. LM could be given by

$$
x_{n+1}=r x_{n}\left(1-x_{n}\right)
$$

where $x_{n} \in[0,1]$ is value of $x$ in current iteration of map, $x_{n+1}$ is its value in next iteration, $n$ represents sequential number of iteration and $r \in[0,4)$ denotes parameter of the map. The value of $x_{0}$ is called an initial value and set of values of $x_{n}$ is known as iterations of LM.

The interesting property of LM is its behavior. For values of $r \in[3.56995,4)$ it is described as chaotic as the following iterations do not clearly converge to any value and they do not show signs of periodicity $[2,6]$. Later, the research concerning dynamical degradation of chaos denied these claims and suggested that calculations with finite precision could lead to periodic behavior of chaotic maps [7]. This assumption is used in design phase of image encryption algorithms which use precision at least of $10^{-14}$ to ensure sufficient values of period.

LM suffers from several drawbacks. Probably the most notable is so-called transient period. This period occurs when first iterations are computed. Values of these iterations could be predictable and therefore they could not be used for encryption. For overcoming this problem, some of the first iterations are thrown away. Usually the size of transient period is set as a power of 10 (eg 100 or 1000 iterations). An example of this phenomenon is shown in Fig. 2, where first 150 iterations of LM with $x_{0}$ set to 0.5 and $r$ equal to $4-10^{-14}$ are displayed. The transient period is most notable in first twenty iterations. Patterns occurring in following iterations seem to be similar, but after certain amount of iterations, they start to differ significantly.

Another drawback of chaotic maps used for diffusion of pixel intensities is the possibility of phase space reconstruction. If the attacker is able to extract values of some iterations used during encryption, he may be able to estimate their properties or even values of generated PRS. Fundamental theory for this approach was provided by Takens in [8] and Sauer, Yorke and Casdagli [9]. A brief summary of time delay method is given by Kliková and Raidl [10].

In general, the phase space reconstruction is a problem for all chaotic encryption algorithms where the generated PRS directly influences produced ciphertext. This is the case for proposal by Pareek, Patidar and Sud [11], where a set of chaotic maps was applied depending on chosen key. Iterates of these maps were then used in various calculations. Lui and Wang [12] generate the key stream by piecewise linear map whose iterations are altered by another map. Hence the phase space reconstruction of the second map could reveal values leading to generated key stream. Wang, Teng and Qin [13] directly add values produced by calculations with iterations of chaotic map to image after confusion. Another algorithm which uses generated iterations directly for operation of bitwise XOR is described by Wang and Guo [14].

Several remedies were proposed in order to suppress the possibility of successful phase space reconstruction attack. Zhu [15] tries to improve computational complexity of the attack by combining iterations of four dimensional chaotic map during diffusion. Murillo-Escobar et al [16] also rise the complexity of attack by using sum of 50 iterations of LM in one step of their diffusion algorithm. Other approach is presented by Tong et al [17], where a new chaotic map based on modified version of LM is designed and its properties are described.

\section{Proposed solution}

In our approach, we tried to utilize the fact that iterating the map (1) is quite simple operation and hence it is not difficult by means of computational complexity. Therefore, if the amount of computed iterations would be greater, the difference in processing time could be negligible for users. We provide Tab. 1 for illustration of processing times, which are needed for calculations of different amount of iterations $N_{i}$ by (1). Presented values are arithmetic means of 100 repeated measurements, the value of $x_{0}$ was equal to 0.5 , parameter $r$ was set to $4-10^{-14}$ and first 100 iterations were discarded for overcoming the transient period. Used PC had $2.5 \mathrm{GHz}$ 

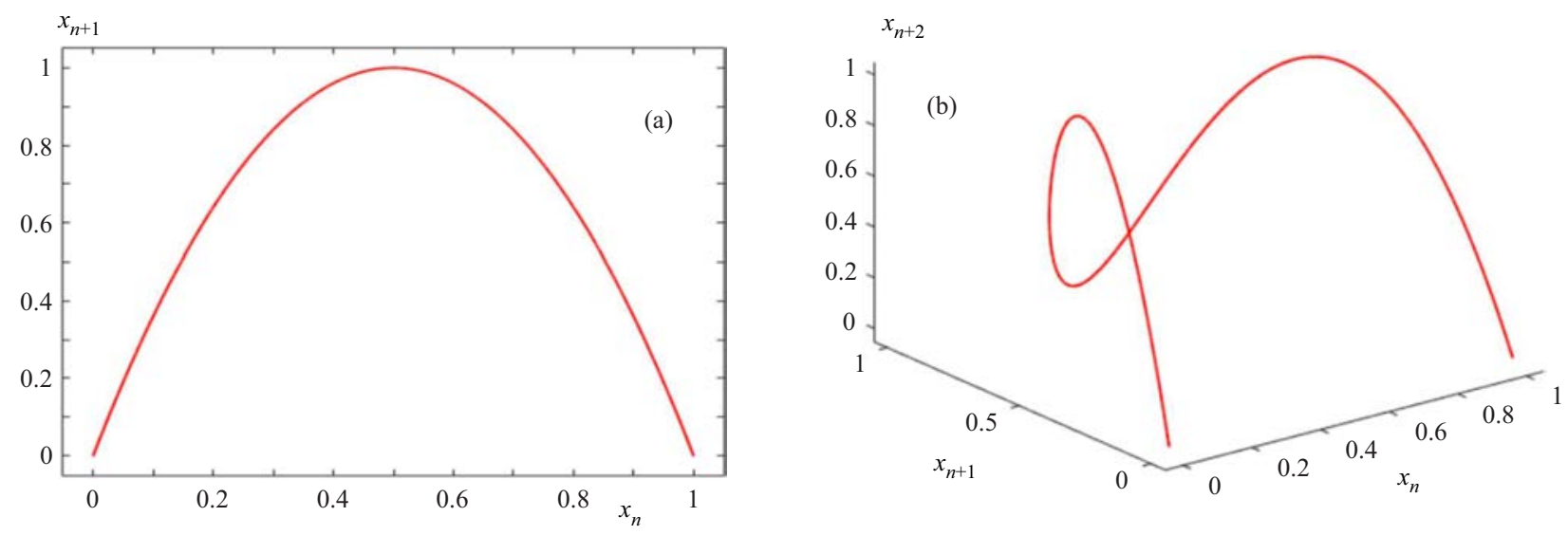

Fig. 3. Poincaré plots for logistic map
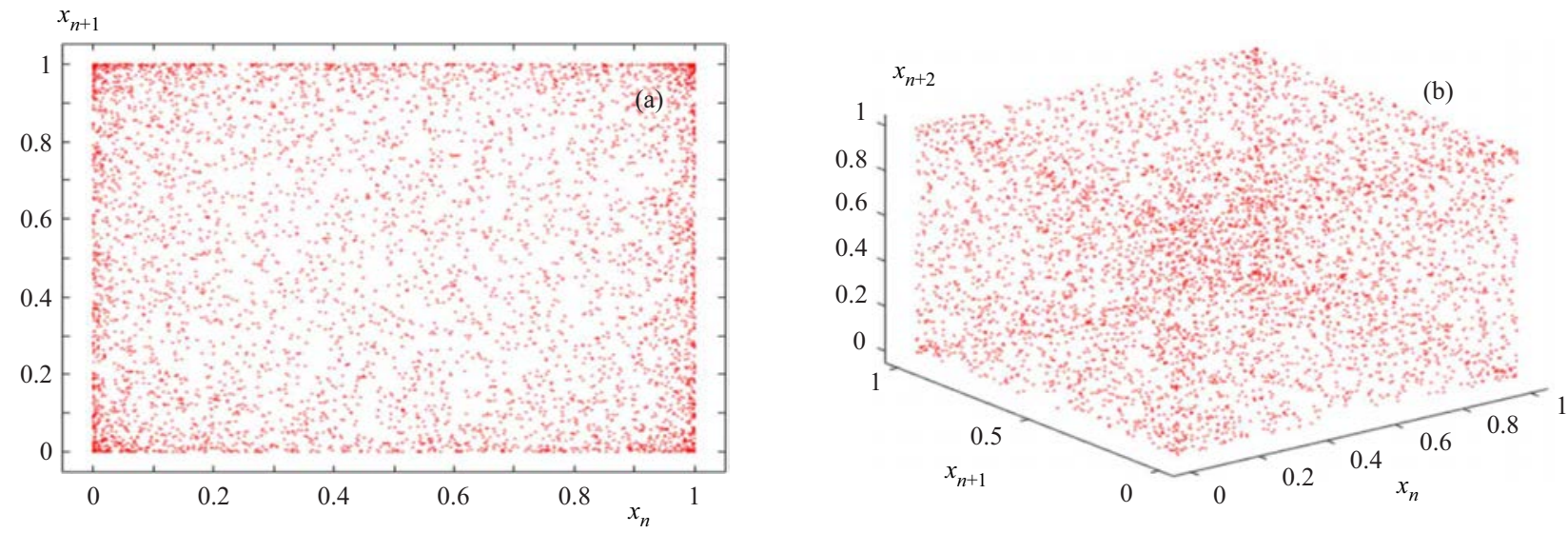

Fig. 4. Examples of Poincaré plots for logistic map with skipped iterations

Table 1. Processing time for iterating logistic map (1): number of iterations $N_{\mathrm{i}}$ and computational time

\begin{tabular}{cccc}
\hline$N_{\mathrm{i}}$ & time $(\mathrm{ms})$ & $N_{\mathrm{i}}$ & time $(\mathrm{ms})$ \\
\hline 100 & 0.005 & $10^{5}$ & 1.33 \\
1000 & 0.016 & $10^{6}$ & 15.72 \\
10000 & 0.147 & $10^{7}$ & 162.59 \\
\hline
\end{tabular}

CPU, 12 gigabytes of RAM and the simulations were run in MATLAB R2015a.

In our proposal, the greater amount of iterations of LM is used for establishing resistance against phase space reconstruction attack. This is possible by selecting iterations, which would be discarded and hence not used for purposes of encryption. If the attackers try to guess the value of last iterate of LM and use it for reconstruction of the phase space, any skipped iterate would result in inaccurate evaluation of following values. This leads to incorrectly decrypted image.

The properties of map regarding resistance to phase space reconstruction could be shown in a Poincaré plot. These plots use values of consecutive iterations of a map as coordinates of plotted points. Two and three dimensional Poincaré plots for LM given by (1) are shown in
Fig. 3. The value of $x_{0}$ was fixed at 0.5 , while $r$ was equal to $4-10^{-14}$. First 100 iterations were discarded to suppress the effects of transient period and subsequent 10000 iterations were used for the plots.

Poincaré plots of LM explain the difficulty behind evaluation of previously generated iterations. Consider finding out value of only one iterate. The plots show that there are two possible values leading to one value in next iteration of LM. If the amount of evaluated iterations rises to num, the number of possible sequences is then $2^{\text {num }}$.

As it was already mentioned, our solution discards some of iterations. These are chosen by keys entered by user. Therefore the dependencies between consecutive iterations are broken according to selected keys. This fact is also reflected by Poincaré plots which are shown in Fig. 4. The plots display the same amount of points as those in previous figure. Plots were obtained for PRS used during diffusion stage of encryption which used image lena. This image was grayscale with resolution of $512 \times 512$ pixels. The selected diffusion key was 0xF0BC940120118097B147F0E35A0EF23E. Details about encryption algorithm are described in following subparagraphs.

The second set of Poincaré plots shows that phase space reconstruction needs to evaluate more possible 


\begin{tabular}{llcccccc}
\hline & $\operatorname{pos}_{s k}(1: 6)$ & 5 & 10 & 15 & 3 & 2 & 1 \\
Step 2 & $\operatorname{size}_{s k}(1: 6)$ & 7 & 13 & 15 & 1 & 7 & 6 \\
& $\operatorname{pos}_{s k}(7: 12)$ & 11 & 13 & 3 & 9 & 2 & 3 \\
& $\operatorname{size}_{s k}(7: 12)$ & 15 & 4 & 2 & 14 & 12 & 1 \\
& $\operatorname{pos}_{s k}(1: 6)$ & 5 & 10 & 15 & 3 & 2 & 1 \\
Step 3 & $\operatorname{size}_{s k}(1: 6)$ & 7 & 13 & 15 & 1 & 7 & 6 \\
& $\operatorname{pos}_{s k}(7: 12)$ & 11 & 13 & 19 & 9 & 18 & 35 \\
& $\operatorname{size}_{s k}(7: 12)$ & 15 & 4 & 18 & 14 & 28 & 34 \\
\hline \hline
\end{tabular}

Fig. 5. Generation of unique positions and sizes for iterate skipping

points, which lead to a known iterate. The number of these points directly depends on keys selected by user. If these keys would be revealed, the whole algorithm would have similar drawbacks as approaches described in paragraph 2 .

\subsection{Key related skipping of iterations}

The discarding of iterations of LM (1) which depends on selected key is done by following algorithm:

Inputs: 12 or 16 byte key $K$, length of sequence to be generated $l e n_{\text {seq }}$, parameter $r \in[3.99,4)$

Output: sequence of iterations $s e q_{s k}$

Step 1: The length of key $L_{\text {key }}$ is determined. Then elements of key $K$ are decomposed into corresponding binary values $K_{\text {bin }}$ by means of little endian ordering scheme (the least significant bit is the first).

Step 2: Binary values of $K_{\text {bin }}$ are split and processed to vectors which determine positions where iterations are skipped $\operatorname{pos}_{s k}$ and amount of the skipped iterations size $e_{\text {k }}$

$$
\begin{gathered}
\operatorname{pos}_{s k}(i)=1+\sum_{b=1}^{4} 2^{b-1} K_{\mathrm{bin}}(i, b) \\
\operatorname{size}_{s k}(i)=1+\sum_{b=1}^{4} 2^{b-1} K_{\mathrm{bin}}(i, b+4)
\end{gathered}
$$

where $i=1,2, \ldots, L_{k e y}$.

Step 3: The resulting values of positions for iterate skipping pos $_{s k}$ are scanned for purpose of modifying not unique values. These positions and their corresponding amounts of skipped iterations $s i z e_{s k}$ are treated by consecutive additions of 16 until all the positions are unique.

Step 4: The value of initial amount of skipped iterations size $_{s k}(0)$ is computed. As this number needs to be unique for every possible user key, it is calculated by

$$
\text { size }_{s k}(0)=\sum_{i=1}^{L_{\mathrm{key}}} i \operatorname{pos}_{s k}(i) \operatorname{size}_{s k}(i) \text {. }
$$

Step 5: Total number of necessary iterations num $_{i t}$ is calculated as

$$
n_{i t}=\operatorname{len}_{\mathrm{seq}}+\operatorname{size}_{s k}(0)+\sum_{i=1}^{L_{\mathrm{key}}}\left\lfloor\frac{\operatorname{len}_{\mathrm{seq}}}{\operatorname{pos}_{s k}(i)}\right\rfloor \operatorname{size}_{s k}(i) \text {. }
$$

Step 6: Equation of LM is iterated for num $_{\mathrm{it}}$ times with $x_{0}$ equal to 0.5 and parameter $r$. The resulting sequence is denoted as $s e q_{i t}$. At this point, the $n_{u} m_{\text {it }}$ is not increased for suppressing the transient period, because $s_{i z e_{s k}}(0)$ is large enough to be used for this purpose.

Step 7: Each element of sequence $s e q_{\text {it }}$ is scanned. If its sequential number and one of positions from $\operatorname{pos}_{s k}(i)$ produces modulus of 0 , successive size $_{s k}(i)$ iterations are discarded and therefore not used during encryption or decryption. If the current element is not skipped, it is copied to sequence $s e q_{s k}$.

Effects of some steps of proposed algorithm are illustrated in Fig. 5. Here, $r$ was set as 3.99210459670052, $l e n_{\text {seq }}$ was equal to 262144 and the key $K$ with length of 12 bytes had value of $0 \times 64 C 9 E E 026150 E A 3 C 12 D 8 B 102$.

Please note that underlining in Fig. 5 indicates positions and sizes of iterate skips which were changed in order to be unique.

Our proposal encrypts and decrypts images with arbitrary resolution and various colour depths such as 8 or 24 bits/pixel. The encryption uses confusion prior to diffusion and decryption applies these stages in opposite order. Algorithms described in the following are intended for usage during encryption, the decryption alternatives are analogous but have several changes mentioned further.

\subsection{Confusion}

Our approach performs confusion by following algorithm:

Inputs: 16 byte key $K_{\text {conf }}$, plaintext image $P$

Output: matrix or vector with values after confusion $C_{\text {vec }}$

Step 1: Height $h$, width $w$ and colour depth $d$ of the plaintext image $P$ are determined.

Step 2: The key $K_{\text {conf }}$ is divided into two parts. First four bytes are copied into vector $r_{\text {add }}$ and the other 12 bytes are passed to vector $s k_{\text {conf }}$. The first vector is used for modification of $r_{\text {conf }}$ used for iterating the map (1) and $s k_{\text {conf }}$ is utilized for generating positions and sizes of iterate shifting.

Step 3: Value of parameter $r_{\text {conf }}$ is calculated as

$$
r_{\text {conf }}=3.99+\frac{1}{10^{2} 2^{32}} \sum_{i=1}^{4} 2^{8(i-1)} r_{\text {add }}(i)
$$

where constants of $10^{2}$ and $2^{32}$ are used for ensuring that $r_{\text {conf }} \in[3.99,4)$ also after additions of elements of $r_{\text {add }}$. The precision used during these computations is $10^{-14}$.

Step 4: Sequence of iterations $s e q_{\text {conf }}$ is generated by skipping algorithm with parameters $r_{\text {conf }}, s k_{\text {conf }}$ and $l e n_{\text {seq }}$ equal to $h w$. 


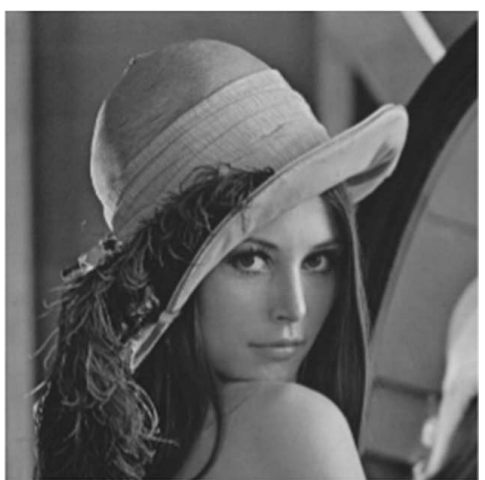

plaintext image

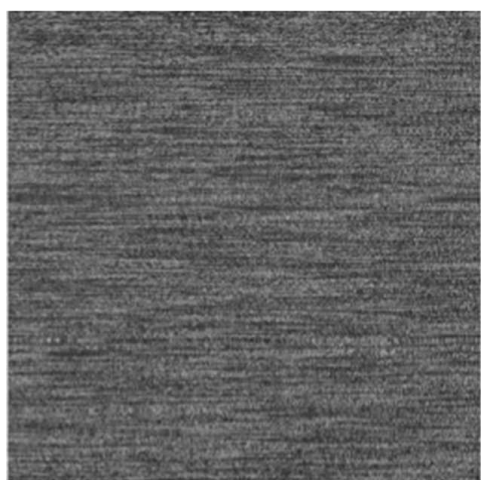

image after confusion

Fig. 6. Illustration of effects of confusion algorithm

Step 5: Elements of $s e q_{\text {conf }}$ are split into two subsequences. First $h$ elements are passed to sequence $s h_{\text {cols }}$ and the other $w$ elements are copied to sequence $s h_{\text {rows }}$. These sequences are then quantized by (6) and (7). Results of quantization are stored in vectors $s h_{\mathrm{colsQ}}$ and $s h_{\text {rowsQ }}$

$$
\begin{aligned}
s h_{\mathrm{colsQ}}(i) & =\left\lfloor s h_{\mathrm{cols}}(i)(h-1)\right\rfloor, \\
s h_{\mathrm{rowsQ}}(j) & =\left\lfloor\operatorname{sh}_{\mathrm{rows}}(j)(w-1)\right\rfloor
\end{aligned}
$$

where $i=1,2, \ldots, h$ and $j=1,2, \ldots, w$.

Step 6: Pixels in columns of plaintext image $P$ are shuffled by using $s h_{\text {colsQ }}(8)$, (9). The shuffling is done in all colour planes. Resulting pixel columns are copied to matrix

$$
\begin{aligned}
& C_{1}\left(l^{\prime}, k, t\right)=P(l, k, t), \\
& l^{\prime}=1+\left(l+s h_{\text {cols } Q}(k)(\bmod h)\right)
\end{aligned}
$$

where $l, l^{\prime}$ are old and new indices of image rows, $k$ is index of image columns and $t \in\{1,2, \ldots, d / 8\}$ is index of colour plane.

Step 7: Pixels in rows of matrix $C_{1}$ are shuffled by using $s h_{\text {rows }}(10),(11)$. The shuffling is done in all colour planes. Resulting pixel rows are copied to matrix

$$
\begin{aligned}
& C_{2}\left(l^{\prime}, k^{\prime}, t\right)=C_{1}\left(l^{\prime}, k, t\right), \\
& k^{\prime}=1+\left(k+\operatorname{sh}_{\text {rows } Q}\left(l^{\prime}\right)(\bmod w)\right)
\end{aligned}
$$

where $k^{\prime}$ is new index of image columns.

Step 8: Elements from matrix $C_{2}$ are passed to matrix $C_{\text {vec }}$ with $d / 8$ rows and $h w$ columns. Hence, each row of this matrix contains shuffled pixels from one colour plane. The pixels are scanned as columns from top left corner to the bottom right one. If plaintext image $P$ is grayscale, the $C_{\text {vec }}$ is vector.

Effects of proposed confusion algorithm are displayed in Fig. 6. Used plaintext image lena was grayscale with resolution of $512 \times 512$ pixels. The value of confusion key was selected as 0x58AFE03564C9EE026150EA3C12D8B102. For purposes of this example, the vector $C_{\text {vec }}$ was reshaped to a matrix with height and width of the plaintext image.

\subsection{Diffusion}

The diffusion is applied by following these steps:

Inputs: 16 byte key $K_{\text {diff }}$, matrix with values after confusion $C_{\mathrm{vec}}$, height $h$ and width $w$ and colour depth $d$ of the plaintext image $P$

Output: encrypted image $E$

Step 1: Sequence of iterations $s e q_{\text {diff }}$ is generated by skipping algorithm with $r_{\text {diff }}=4-10^{-14}$ and parameters $K_{\text {diff }}$ and $l e n_{\text {seq }}$ equal to $\lceil 2 h w d / 8 / 7\rceil$. As the used precision is $10^{-14}$, the generated iterations have $14 \mathrm{dec}-$ imal places.

Step 2: Each element of $s e q_{\text {diff }}$ is split to seven parts. These parts are stored in matrix $s e q_{\operatorname{diff} M}$

$$
\operatorname{seq}_{\mathrm{diff} M}(j, i)=\left\lfloor 10^{2 j} \operatorname{seq}_{\mathrm{diff}}(i)(\bmod 100)\right\rfloor
$$

where $i=1,2, \ldots$, len $_{\text {seq }}$ and $j=1,2, \ldots, 7$.

Step 3: Matrix $s e q_{\mathrm{diff} M}$ is rearranged to vector $s e q_{\mathrm{diff} V}$ by scanning columns of $s e q_{\operatorname{diff} M}$ starting with top left corner continuing to the bottom right one. Then elements of $s e q_{\mathrm{diff} V}$ are quantized and copied to vector $s e q_{\text {diff } Q}$

$$
\operatorname{seq}_{\mathrm{diff} Q}(i)=\operatorname{round}\left(255 \frac{\operatorname{seq} q_{\mathrm{diff} V}(i)+1}{100}\right)
$$

where $i=1,2, \ldots, 7$ len $_{\text {seq }}$. The values of $s e q_{\mathrm{diff} V}$ use addition of one because (12) produces values from set $\{0,1, \ldots, 99\}$.

Step 4: First iteration of diffusion takes place, separately in each colour plane. Results of ciphertext chaining done by (14) are passed to vector $c h_{1}$. Then elements of $s e q_{\text {diff } Q}$ modify these values by (15). Computed values representing image pixels after first iteration of diffusion are stored in matrix $D_{\mathrm{vec}}$.

$c h_{1}(m, i)=$

$\begin{cases}C_{\mathrm{vec}}(t, 1)+C_{\mathrm{vec}}(t, h w)(\bmod 256) & \text { if } i=1, \\ C_{\mathrm{vec}}(t, i)+c h_{1}(t, i-1)(\bmod 256) & \text { otherwise, }\end{cases}$

$$
D_{\mathrm{vec}}(t, i)=c h_{1}(t, i) \oplus s e q_{\mathrm{diff} Q}(i+h w(t-1))
$$




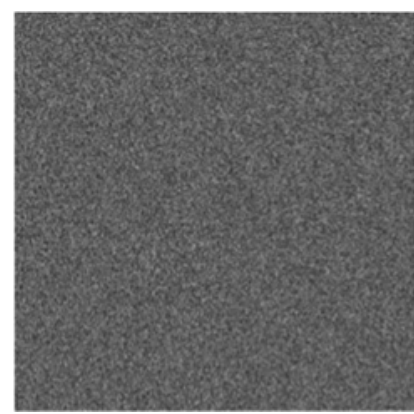

image after confusion

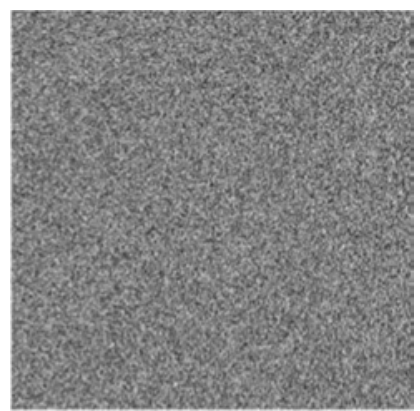

image after diffusion
Fig. 7. Demonstration of properties of diffusion algorithm

where $i=1,2, \ldots, 7$ len $_{\text {seq }}, t \in\{1,2, \ldots, d / 8\}$ is the index of colour plane and $\oplus$ is the operator of bitwise XOR.

Step 5: Second iteration of diffusion takes place, separately in each colour plane. Results of ciphertext chaining done by (16) are passed to vector $c h_{2}$. Then elements of $s e q_{\text {diffQ }}$ modify these values by (17). Computed values representing image pixels after first iteration of diffusion are stored in matrix $E_{\mathrm{vec}}$.

$$
\begin{aligned}
& \operatorname{ch}_{2}(t, i)= \\
& \begin{cases}D_{\mathrm{vec}}(t, 1)+D_{\mathrm{vec}}(t, h w)(\bmod 256) & \text { if } i=1 \\
D_{\mathrm{vec}}(t, i)+c h_{2}(t, i-1)(\bmod 256) & \text { otherwise }\end{cases}
\end{aligned}
$$

$$
E_{\mathrm{vec}}(t, i)=\operatorname{ch}_{2}(t, i) \oplus \operatorname{seq}_{\mathrm{diff} Q}(i+h w(d / 8+(t-1))) .
$$

Step 6: Elements of matrix $E_{\text {vec }}$ are passed to matrix $E$ with $h$ rows, $w$ columns and $d / 8$ colour planes. The elements are copied separately for each colour plane, they are scanned as columns from top left corner to the bottom right one. Matrix $E$ represents encrypted version of plaintext image $P$.

Some differences between confusion and diffusion algorithms should be pointed out. Firstly, the diffusion is done in two steps. This is necessary for creating dependencies between higher amount of image pixels. Consider following case: there are two identical plaintext images $P_{1}$ and $P_{2}$. A difference with minimal size is introduced in one pixel located in bottom right corner of $P_{2}$ (intensity of the pixel is either increased or decreased). If encryption algorithm uses only one iteration of diffusion and therefore one iteration of chaining, the difference will not spread to successive image pixels, because it is located at pixel which is scanned as the last. Second iteration of diffusion uses this modified value for computing value representing first pixel, so in this case, the resulting encrypted images $E_{1}$ and $E_{2}$ should be different.

Secondly, there is a contrast between lengths of key used for iterate skipping during confusion and diffusion. This is caused by different objectives of these stages. While our confusion step shuffles large amount of plaintext image pixels, the diffusion "only" introduces chaotic behavior to results of ciphertext chaining. Therefore, it is important to ensure that sequences generated for confusion are more diverse than those calculated for diffusion.

Finally, diffusion stage utilizes one iterate of LM for modification of seven pixel intensities, while confusion uses one iterate for shifting each column, or row of image pixels. Also the confusion step shuffles image pixels in similar manner in each colour plane, while diffusion uses different values for each colour plane of the processed image.

Impact of our diffusion stage is shown in Fig. 7. The left image was created by applying confusion to plaintext image lena (see Fig. 6 for details). Chosen diffusion key had value of $0 \mathrm{xF} 0 B C 940120118097 B 147 F 0 E 35 A 0 E F 23 E$.

\subsection{Alterations used for decryption}

As it was already mentioned, the diffusion is used prior to confusion in case of image decryption. The sequences used for these two operations are created similarly as during encryption. Then the two iterations of diffusion are removed by performing bitwise XOR and inverse version of ciphertext chaining which uses subtraction instead of addition. Confusion stage obtains original positions of image pixels by applying reverse shifts in columns of image before reverse shifts in image rows.

\section{Experimental results}

All following experiments were performed in MATLAB R2015a running in Windows 10 OS on PC with $2.5 \mathrm{GHz}$ CPU and 12 gigabytes of RAM.

Table 2. Properties of Used Images

\begin{tabular}{|c|c|c|}
\hline$K$ & & value \\
\hline 1 & $\begin{array}{l}K_{\text {conf }} \\
K_{\text {diff }}\end{array}$ & $\begin{array}{l}\text { 0x58AFE03564C9EE026150EA3C12D8B102 } \\
\text { 0xF0BC940120118097B147F0E35A0EF23E }\end{array}$ \\
\hline 2 & $\begin{array}{l}K_{\text {conf }} \\
K_{\text {diff }}\end{array}$ & $\begin{array}{l}\text { 0x58AEE03564C9EE026150EA3C12D8B102 } \\
\text { 0xF0BC940120118097B147F0E35A0EF23E }\end{array}$ \\
\hline 3 & $\begin{array}{l}K_{\text {conf }} \\
K_{\text {diff }}\end{array}$ & $\begin{array}{l}\text { 0x58AFE03564C9EE126150EA3C12D8B102 } \\
\text { 0xF0BC940120118097B147F0E35A0EF23E }\end{array}$ \\
\hline 4 & $\begin{array}{l}K_{\text {conf }} \\
K_{\text {diff }}\end{array}$ & $\begin{array}{l}\text { 0x58AFE03564C9EE026150EA3C12D8B102 } \\
\text { 0xF0BC940120118097B147F0E36A 0EF23E }\end{array}$ \\
\hline
\end{tabular}

\begin{tabular}{lllll}
\hline \multirow{2}{*}{ property } & \multicolumn{4}{c}{ image } \\
& Lena & Peppers & Chaos & Black \\
\hline resolution & 512 & 512 & 512 & 512 \\
$w \times h(\mathrm{px})$ & $\times 512$ & $\times 512$ & $\times 256$ & $\times 256$ \\
$\begin{array}{l}\text { colour depth } \\
d(\mathrm{bit} / \mathrm{px})\end{array}$ & 8 & 24 & 8 & 8 \\
\hline
\end{tabular}

Table 3. Used key values 


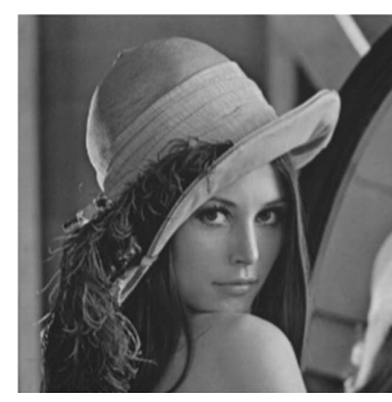

lena

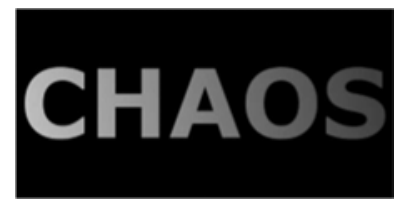

chaos

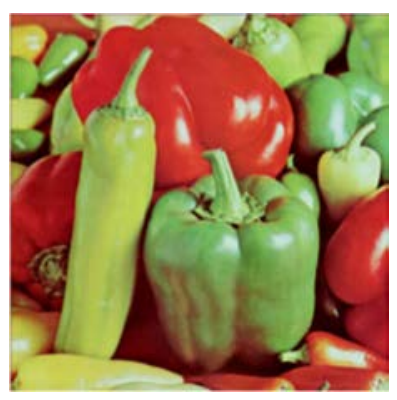

peppers

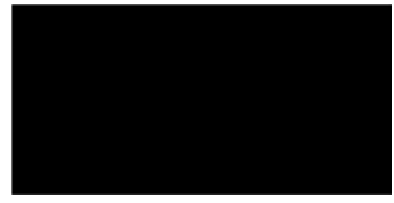

black
Fig. 8. Set of images used during experiments

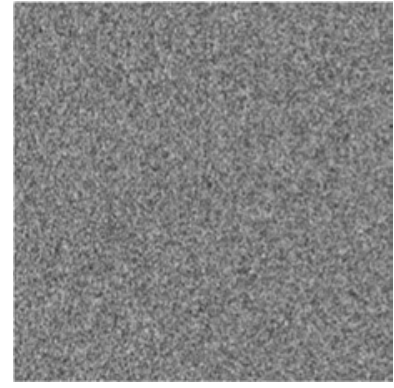

lena

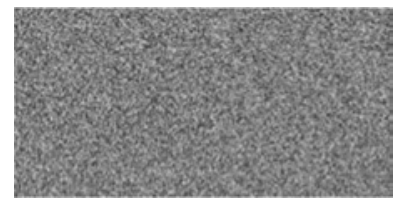

chaos

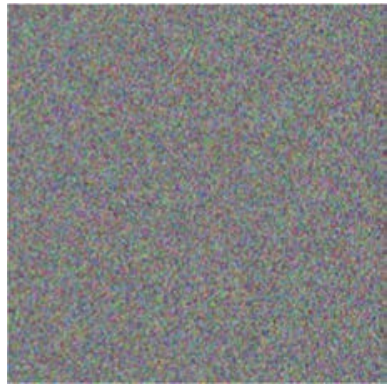

peppers

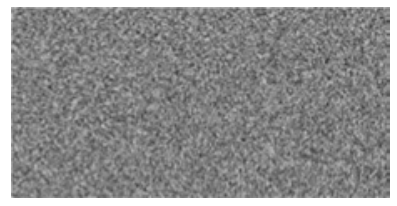

black
Fig. 9. Encrypted versions of used images

The set of used images is shown in Fig. 8. Their parameters are included in Table 2. Keys utilized during experiments are provided in Table 3. Differences between keys are indicated by underlined characters.

The differences between keys were made intentionally on certain places. $K_{2}$ produces different $r_{\text {conf }}$ than $K_{1}$ and usage of $K_{3}$ or $K_{4}$ instead of $K_{1}$ results in different sequences of iterations generated during confusion or diffusion stage.

Encrypted versions of images from Fig. 8 are displayed in Fig. 9. Key used for this purpose was $K_{1}$.

\subsection{Size of key space and analysis of key sensitivity}

Key space includes all possible combinations of keys. Because the confusion key $K_{\text {conf }}$ and diffusion key $K_{\text {diff }}$ do not depend on each other, the size of key space could be computed as num $_{\text {keys }}=256^{16} \times 256^{16}=2^{128+128}=$ $2^{256}$.
The dependence on used key is illustrated in Fig. 10. Left column of this figure shows results of encryption of lena by keys $K_{1}, K_{3}$ and $K_{4}$. Right column contains examples of difference image and decryptions by correct and incorrect key.

\subsection{Statistical attacks}

This kind of attacks tries to search for dependencies between plaintext images and their corresponding encrypted versions. In order to prevent possibility of statistical attacks, the diffusion stage should disturb these relations.

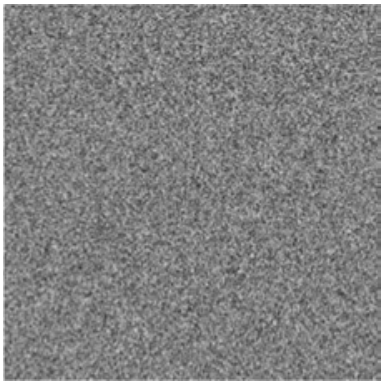

encrypted by $K_{1}$

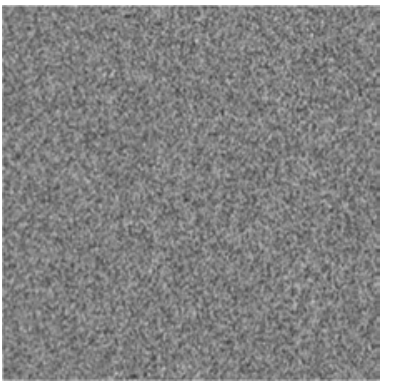

encrypted by $K_{3}$

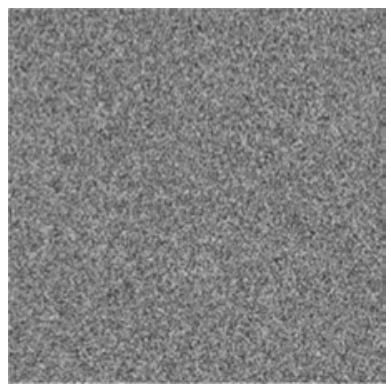

encrypted by $K_{4}$

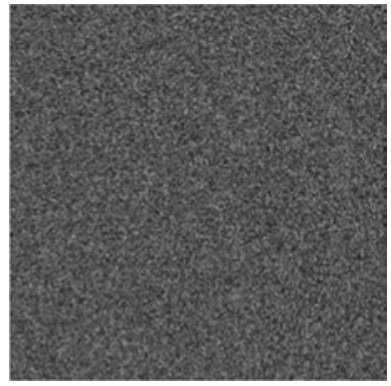

difference between images encrypted by $K_{3}$ and $K_{4}$

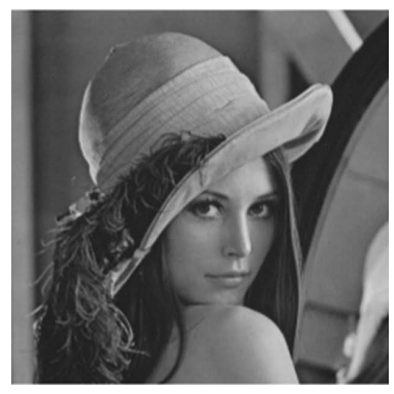

encrypted by $K_{1}$ and decrypted by $K_{1}$

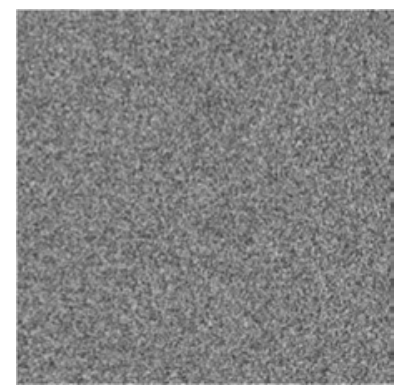

encrypted by $K_{1}$ and decrypted by $K_{4}$
Fig. 10. An example of key sensitivity

The resistance against statistical attacks could be evaluated by various measures, such as shape of histograms, correlation diagrams, correlation coefficients or the value of entropy.

Histograms of plaintext image lena and its version encrypted by $K_{1}$ are shown in Fig. 11. The second histogram does not contain so notable peaks as the histogram of plaintext image, which means that small amount of information usable for statistical attacks could be extracted from these histograms. 
Table 4. Computed values of numeric parameters

\begin{tabular}{|c|c|c|c|c|}
\hline $\begin{array}{l}\text { image } \\
\text { and key }\end{array}$ & $\begin{array}{c}\rho_{\text {hor }} \\
(-)\end{array}$ & $\begin{array}{c}\rho_{\mathrm{ver}} \\
(-)\end{array}$ & $\begin{array}{c}\rho_{\text {diag }} \\
(-)\end{array}$ & $\begin{array}{c}H \\
(\mathrm{bits} / \mathrm{px})\end{array}$ \\
\hline & \multicolumn{4}{|c|}{ plain-text images } \\
\hline Lena & 0.9730 & 0.9752 & 0.9562 & 7.2344 \\
\hline Peppers & & & & \\
\hline $\begin{array}{l}(R) \\
(G)\end{array}$ & $\begin{array}{l}0.9536 \\
0.9731\end{array}$ & $\begin{array}{l}0.9642 \\
0.9785\end{array}$ & $\begin{array}{l}0.9446 \\
0.9609\end{array}$ & $\begin{array}{l}7.3388 \\
7.4962\end{array}$ \\
\hline$(B)$ & 0.9649 & 0.9661 & 0.9339 & 7.0583 \\
\hline Chaos & 0.9748 & 0.9767 & 0.9469 & 2.0180 \\
\hline \multirow[t]{2}{*}{ Black } & \multicolumn{3}{|c|}{ division by zero } & 0 \\
\hline & \multicolumn{4}{|c|}{ encrypted images } \\
\hline Lena, $K_{1}$ & 0.0054 & 0.0017 & 0.0036 & 7.9993 \\
\hline Lena, $K_{2}$ & -0.0019 & -0.00169 & 0.0031 & 7.9993 \\
\hline \multicolumn{5}{|l|}{ Peppers } \\
\hline$(R), K_{1}$ & -0.0057 & -0.0018 & 0.0006 & 7.9993 \\
\hline$(R), K_{2}$ & -0.0023 & -0.0014 & -0.0038 & 7.9993 \\
\hline$(G), K_{1}$ & 0.0053 & 0.0052 & -0.0008 & 7.9992 \\
\hline$(G), K_{3}$ & -0.0027 & -0.0016 & 0.0049 & 7.9992 \\
\hline$(B), K_{1}$ & -0.0006 & -0.0033 & 0.0025 & 7.9984 \\
\hline$(B), K_{4}$ & 0.0006 & -0.0012 & 0.0035 & 7.9986 \\
\hline \multicolumn{5}{|l|}{ Chaos } \\
\hline$K_{1}$ & 0.0000 & -0.0041 & -0.0035 & 7.9986 \\
\hline$K_{3}$ & 0.0059 & 0.0017 & 0.0014 & 7.9987 \\
\hline \multicolumn{5}{|l|}{ Black } \\
\hline$K_{1}$ & -0.0045 & -0.0066 & 0.0001 & 7.9986 \\
\hline$K_{4}$ & -0.0008 & 0.0020 & 0.0068 & 7.9987 \\
\hline
\end{tabular}

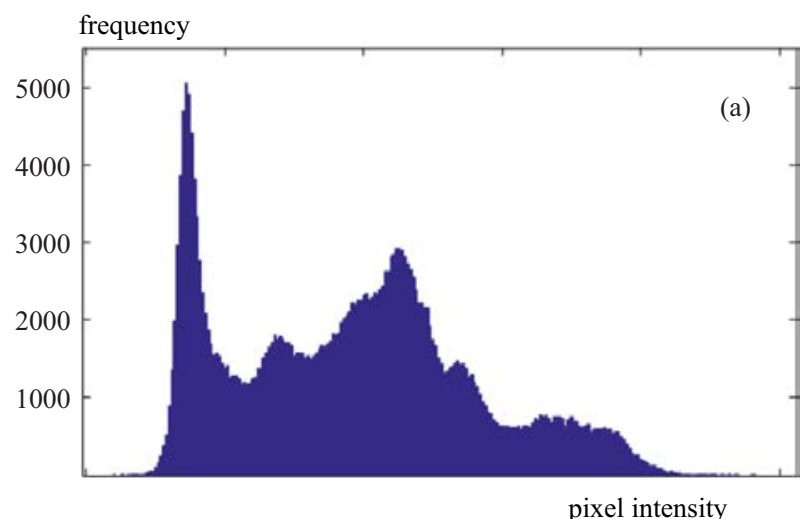

(b)

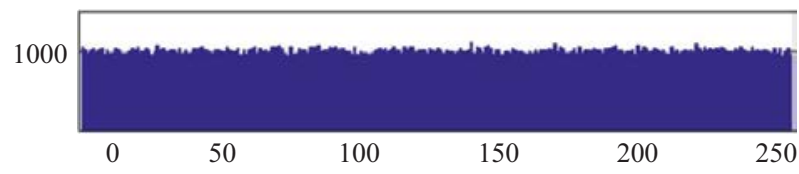

Fig. 11. Comparison of image histograms: (a) - plain-text image, (b) - encrypted by $K_{1}$

Correlation diagrams display similarity between adjacent image pixels. There are three possible kinds of pixel adjacency: horizontal, vertical or diagonal one. The di- agrams for 1000 randomly chosen pairs of horizontally adjacent pixels from plaintext image lena and its version encrypted by $K_{1}$ are shown in Fig. 12. As the intensities of adjacent pixels in plaintext image are similar, the diagram contains many points which lie near line $y=x$. These relations are violated by diffusion stage and therefore the diagram of encrypted image shows more uniform distribution of plotted points.

Correlation coefficients $\rho$ provide numeric values that represent correlation among adjacent pixels in image. They are computed for horizontally, vertically and diagonally adjacent pairs of pixels, separately in each colour plane. Computations of correlation coefficients use following equations

$$
\begin{gathered}
\rho=\frac{\operatorname{cov}_{P, E}}{\sqrt{\sigma_{P}^{2} \sigma_{E}^{2}}}[-], \\
\operatorname{cov}_{P, E}=\sum_{l=1}^{h} \sum_{k=1}^{w}(P(l, k)-\bar{P})(E(l, k)-\bar{E}), \\
\sigma_{I}^{2}=\sum_{l=1}^{h} \sum_{k=1}^{w}(I(l, k)-\bar{I})^{2}, \\
\bar{I}=\frac{1}{h w} \sum_{l=1}^{h} \sum_{k=1}^{w} I(l, k),
\end{gathered}
$$

where $P$ is plaintext image and $E$ is its corresponding encrypted version, $\sigma_{I}^{2}$ is dispersion of arbitrary image $I, l$ and $k$ are pixel coordinates, $h$ and $w$ denote image height and width and $\bar{l}$ represents arithmetic mean of pixel intensities in image $I$.

Entropy $H$ is a measure that was developed for evaluating randomness of data flows [18]. In case of image encryption, the theoretical bound of entropy which would indicate perfectly random image is determined by colour depth of analyzed image. Therefore the bound for grayscale image is 8 bits/pixel. Entropy of true colour images could be calculated separately for each of their colour planes or for all three planes arranged in one sequence of pixels. In this paper, we provide results for each of the colour planes, denoted as $R$ (red), $G$ (green) and $B$ (blue).

Entropy $H$ of image or colour plane with colour depth of $8 \mathrm{bits} /$ pixel could be calculated as

$$
H=-\sum_{i=0}^{255} p(\text { in }) \log _{2}(p(\text { in }))[\text { bits/pixel }]
$$

where $p(i n)$ denotes frequency of pixels with intensity $i n$.

Values of numeric parameters - correlation coefficients $\rho$ and entropy $H$ calculated for various combinations of plaintext images and keys are included in Tab. 4.

There are several remarks about values presented in Tab. 4. First of all, values of correlation coefficients and entropy differ depending on the used key. Some of these differences are quite big (values for black encrypted by $K_{1} v s$ values encrypted by $\left.K_{4}\right)$. The positive thing is 

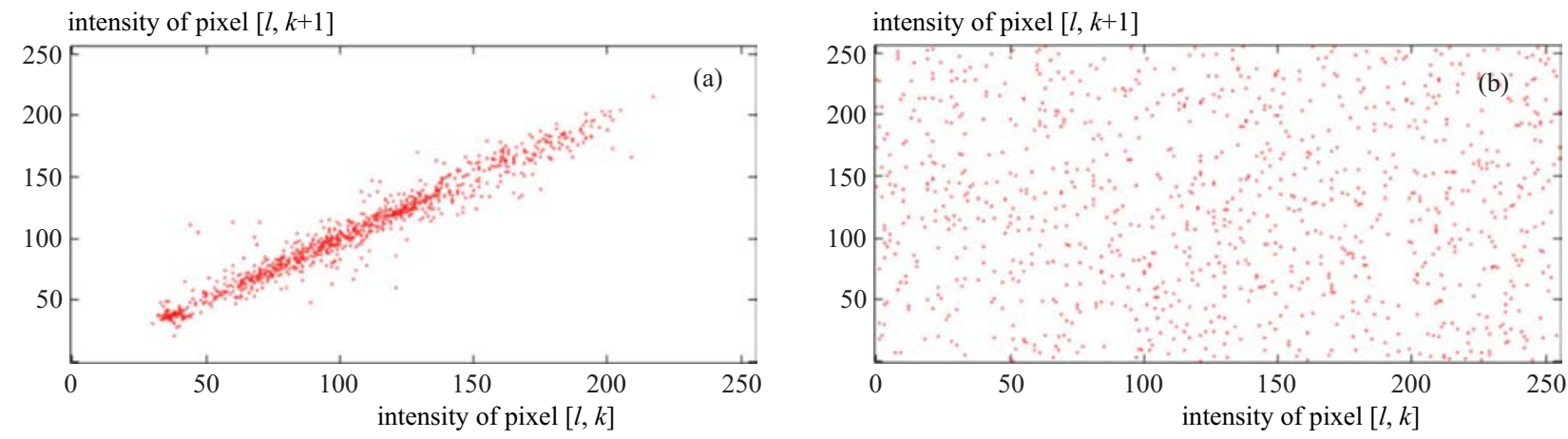

Fig. 12. Correlation diagrams for tested image

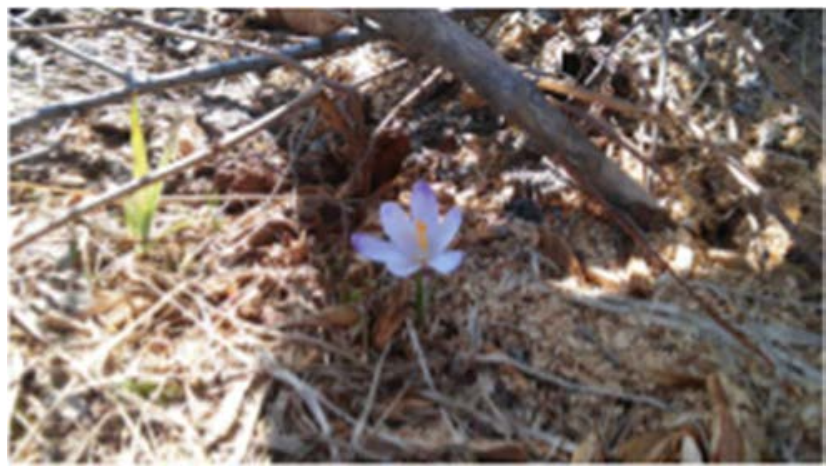

flower

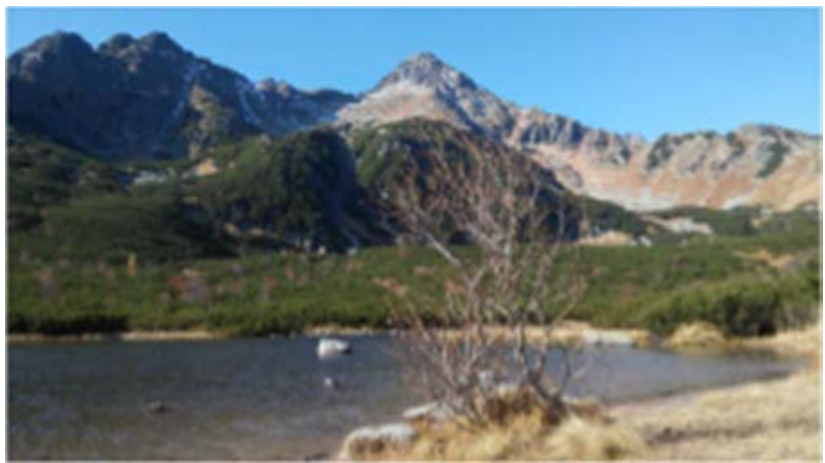

$\operatorname{tarn}$

Fig. 13. Thumbnails of images used for randomness evaluation

Table 5. Achieved values of NPCR and UACI

\begin{tabular}{lcc}
\hline image and key & NPCR & UACI \\
\hline Lena & & \\
$K_{1}$ & 99.8023 & 33.4692 \\
$K_{2}$ & 99.7986 & 33.4747 \\
Peppers & & \\
$(R), K_{1}$ & 99.8200 & 33.4577 \\
$(R), K_{2}$ & 99.7791 & 33.4720 \\
$(G), K_{1}$ & 99.8231 & 33.4632 \\
$(G), K_{3}$ & 99.8014 & 33.4569 \\
$(B), K_{1}$ & 99.8196 & 33.4677 \\
$(B), K_{4}$ & 99.7985 & 33.4763 \\
Chaos & & \\
$K_{1}$ & 99.8156 & 33.4643 \\
$K_{3}$ & 99.8302 & 33.4713 \\
Black & & \\
$K_{1}$ & 99.7942 & 33.4736 \\
$K_{4}$ & 99.7969 & 33.4789 \\
\hline
\end{tabular}

that absolute values of all obtained correlation coefficients for encrypted images are smaller than 0.01. Secondly, the entropy $H$ is greater than 7.998 bits/pixel for all mentioned combinations.

\subsection{Differential attacks}

Differential attacks try to reveal properties of encryption algorithm by performing encryption on images with only small differences. A quite usual approach is the introduction of minimal difference between two plaintext images $P_{1}$ and $P_{2}$ and performing encryption with same parameters which produces images $E_{1}$ and $E_{2}$. This approach is applied also in two parameters that evaluate performance of encryption algorithms [19]. If used plaintext images are true colour, the difference is made only in one of their colour planes.

First of these parameters is NPCR (Number of Pixel Change Ratio) which sums the amount of image pixels with different intensities and divides this sum with total number of pixels in tested image. Hence, NPCR of image or colour plane with colour depth of $8 \mathrm{bits} /$ pixel is given by

$$
\begin{gathered}
\text { NPCR }=\frac{100}{h w} \sum_{l=1}^{h} \sum_{k=1}^{w} \operatorname{Diff}_{\text {mat }}(l, k)[\%], \\
\operatorname{Diff}_{\text {mat }}(l, k)= \begin{cases}0 & \text { if } E_{1}(l, k)=E_{2}(l, k), \\
1 & \text { otherwise }\end{cases}
\end{gathered}
$$

where Dif $f_{\text {mat }}(l, k)$ is difference matrix.

Second parameter, UACI (Unified Average Changing Intensity) considers also the sizes of intensity changes. 
Table 6. Evaluation of randomness for two images

\begin{tabular}{lcccc}
\hline image & \multicolumn{2}{c}{ Flower } & \multicolumn{2}{c}{ Tarn } \\
test & P-value & proportion & P-value & proportion \\
\hline $\begin{array}{l}\text { Frequency (Monobit) } \\
\text { Frequency }\end{array}$ & 0.141256 & $128 / 128$ & 0.484646 & $128 / 128$ \\
within a block $(m=128)$ & 0.862344 & $127 / 128$ & 0.213309 & $127 / 128$ \\
Cumulative sums & & & & \\
(forward) & 0.723129 & $127 / 128$ & 0.082177 & $128 / 128$ \\
(reverse) & 0.772760 & $127 / 128$ & 0.100508 & $127 / 128$ \\
Runs & 0.500934 & $128 / 128$ & 0.364146 & $127 / 128$ \\
Longest run in a block & 0.195163 & $128 / 128$ & 0.97606 & $128 / 128$ \\
Binary matrix rank & 0.922036 & $127 / 128$ & 0.264458 & $127 / 128$ \\
Spectral (FFT) & 0.888137 & $126 / 128$ & 0.110952 & $127 / 128$ \\
Non-overlapping template & & & & \\
(first $P$-value for $m=9)$ & 0.299251 & $127 / 128$ & 0.551026 & $127 / 128$ \\
Overlapping template & & & & \\
(first $P$-value for $m=9)$ & 0.602458 & $127 / 128$ & 0.484646 & $125 / 128$ \\
Maurer's universal test & 0.723129 & $125 / 128$ & 0.900104 & $126 / 128$ \\
Approximate entropy & & & & \\
( $m=10)$ & 0.819544 & $126 / 128$ & 0.875539 & $127 / 128$ \\
Random excursions & & & & \\
(first $P$-value) & 0.559523 & $80 / 80$ & 0.350485 & $82 / 86$ \\
variant (first $P$-value) & 0.484646 & $80 / 80$ & 0.509162 & $84 / 86$ \\
Serial 1 $(m=16)$ & 0.468595 & $126 / 128$ & 0.756476 & $127 / 128$ \\
Serial $2(m=16)$ & 0.452799 & $128 / 128$ & 0.468595 & $128 / 128$ \\
Linear complexity $(M=500)$ & 0.804337 & $124 / 128$ & 0.922036 & $128 / 128$ \\
\hline
\end{tabular}

Value of UACI for image or colour plane with colour depth of 8 bits/pixel is computed by

$$
U A C I=\frac{100}{h w} \sum_{l=1}^{h} \sum_{k=1}^{w} \frac{\left|E_{1}(l, k)-E_{2}(l, k)\right|}{2^{d}-1}[\%]
$$

where $d$ represents colour depth of image or its colour plane.

Computed values of NPCR and UACI for various combinations of plaintext images and keys are shown in Tab. 5. The values are arithmetic means of 100 repeated measurements.

Results shown in Tab. 5 lead to several conclusions. All values of NPCR exceed $99.77 \%$ and UACI achieves at least level of $33.45 \%$. These values depend on the used key, as it was notable also for correlation coefficients and entropy.

\subsection{Evaluation of randomness}

Performance of an encryption algorithm could be measured by several sets of statistical tests. Selected portion of tests used for choosing candidate algorithms for AES [20] was later released as NIST 800-22 suite [21]. We used this set of tests for evaluating performance of proposed algorithm.
The NIST 800-22 suite calculates one $P$-value for each of performed tests. The encrypted images could be considered as strings of random bits if their corresponding $P$-values achieve values greater than chosen significance level $\alpha$. The default value of $\alpha$ is 0.01 [21].

Tests from NIST 800-22 suite require certain amount of data for their calculations. The results of tests are also given as proportion of subsequences which pass the test and total amount of these subsequences (usually set to 100 or 128). NIST recommends length of the subsequences $n$ at least equal to $10^{6}$. Therefore, the minimal size of data used for testing randomness of image encryption algorithm is $100 \times 10^{6} \mathrm{bits}$, which is approximately 11.921 megabytes.

We used two digital images for purposes of testing, both with resolution of $3264 \times 1836$ pixels and colour depth of 24 bits/pixel. Thumbnails of these images are shown in Fig. 13. The images were encrypted by $K_{1}$.

For enabling possibility of repeatable experiments, we present uploaded plaintext images as [22]. These images are provided free of charge for scientific purposes. Binary strings of required length were obtained from encrypted versions of images by decomposition to their colour planes. Pixel intensities of these planes were then scanned as columns of pixels starting from top left corner and continuing to the bottom right one. Finally, each of 
Table 7. Time elapsed during operations and corresponding processing speeds

\begin{tabular}{lcccc}
\hline operation & \multicolumn{2}{c}{ encryption } & \multicolumn{2}{c}{ decryption } \\
\hline $\begin{array}{l}\text { image } \\
\text { and key }\end{array}$ & $\begin{array}{c}\text { time } \\
(\mathrm{ms})\end{array}$ & $\begin{array}{c}v_{\text {proc }} \\
(\mathrm{MB} / \mathrm{s})\end{array}$ & $\begin{array}{c}\text { time } \\
(\mathrm{ms})\end{array}$ & $\begin{array}{c}v_{\text {proc }} \\
(\mathrm{MB} / \mathrm{s})\end{array}$ \\
\hline Lena & & & & \\
$K_{1}$ & 235.008 & 1.06379 & 245.545 & 1.01814 \\
$K_{2}$ & 234.496 & 1.06611 & 245.989 & 1.01631 \\
Peppers & & & & \\
$K_{1}$ & 711.998 & 1.05337 & 739.847 & 1.01372 \\
$K_{2}$ & 713.108 & 1.05174 & 741.713 & 1.01117 \\
Chaos & & & & \\
$K_{1}$ & 115.636 & 1.08098 & 122.399 & 1.02125 \\
$K_{3}$ & 116.169 & 1.07602 & 122.139 & 1.02343 \\
Black & & & & \\
$K_{1}$ & 115.636 & 1.08535 & 122.067 & 1.02402 \\
$K_{4}$ & 116.169 & 1.07981 & 122.239 & 1.02259 \\
\hline
\end{tabular}

these intensities was decomposed to 8 bits by little endian ordering scheme.

Resulting values for tests from NIST 800-22 suite are presented in Tab. 6. The number of subsequences was set to 128 , their length $n$ was selected as $10^{6}$, and the tests used default values of auxiliary variables $(m$ and $M)$. The value of significance level $\alpha$ was left unchanged at 0.01 , so the encrypted image passes tests if $P$-values are greater than 0.01 . The proportions of subsequences which are needed for passing the test are calculated either as 123 of 128 and 76 of 80 or 82 of 86 subsequences for Random excursions tests. More details about used tests and subsequences which are skipped in some of tests are provided in [21].

Results in Tab. 6 illustrate possible differences between $P$-values of various encrypted images. However, the pass proportion and therefore also the result of testing stays similar in case of both used images.

\subsection{Computational Complexity}

The amount of time needed for operations with our algorithm was tested on $\mathrm{PC}$ with $2.5 \mathrm{GHz} \mathrm{CPU}$ and 12 gigabytes of RAM which ran MATLAB R2015a under Windows 10 OS. Values given in Tab. 7 are arithmetic means of 100 repeated measurements.

The processing speed $v_{\text {proc }}$ is computed simply as size of image divided by processing time $t_{\text {proc }}$ given in seconds

$$
v_{\text {proc }}=\frac{h w d}{2^{20} t_{\text {proc }}}(M B / s)
$$

where $h, w$ and $d$ are height, width and colour depth of processed image. Constant of $2^{20}$ represents amount of bits in one megabyte of data (1 megabyte $=1048576$ bits).

Processing speeds presented in Tab. 7 illustrate impact of chosen combination of image and key on the duration of performed operation. All encryption speeds were higher than $1.05 \mathrm{MB} / \mathrm{s}$, while decryption speeds were slightly slower, they barely rose above value of $1 \mathrm{MB} / \mathrm{s}$. The results also imply that higher resolution causes decrease of the processing speeds.

\subsection{Comparison with other approaches}

Comparison of numeric results of various proposals is quite difficult task mainly due to different images used during experiments. Also some papers provide results separately for each colour plane of true colour images, while other papers contain arithmetic means of computed measures. Calculated processing speeds depend on configuration of used PC and also on environment where the experiments were conducted.

Table 8. Comparison of numeric parameters

\begin{tabular}{|c|c|c|c|c|c|}
\hline $\begin{array}{l}\text { reference } \\
\text { or image } \\
\end{array}$ & $\begin{array}{c}\text { colour } \\
\text { plane }\end{array}$ & $\begin{array}{l}\rho_{\text {hor }} \\
(-)\end{array}$ & $\begin{array}{l}\rho_{\mathrm{ver}} \\
(-) \\
\end{array}$ & $\begin{array}{c}\rho_{\text {diag }} \\
(-) \\
\end{array}$ & $\begin{array}{c}H \\
\text { (bits/px) }\end{array}$ \\
\hline Lena256-8 & - & 0.000797 & -0.000823 & 0.001173 & 7.99701 \\
\hline ref. [15] & - & 0.001005 & -0.000850 & 0.000897 & 7.99770 \\
\hline \multirow{3}{*}{$\begin{array}{l}\text { Lena } \\
256-24\end{array}$} & $\mathrm{R}$ & -0.003044 & -0.001364 & 0.002850 & 7.99729 \\
\hline & G & -0.00287 & -0.004494 & 0.006668 & 7.99691 \\
\hline & B & -0.003423 & -0.003736 & -0.005474 & 7.99685 \\
\hline \multirow{3}{*}{$\begin{array}{l}\text { ref. } \\
{[17]}\end{array}$} & $\mathrm{R}$ & 0.003153 & 0.009650 & 0.017851 & \multirow{3}{*}{ 7.999987* } \\
\hline & G & 0.006827 & 0.004191 & 0.012953 & \\
\hline & B & 0.001469 & 0.003467 & 0.009146 & \\
\hline \multirow{3}{*}{$\begin{array}{l}\text { Lena } \\
512-24\end{array}$} & $\mathrm{R}$ & -0.001531 & -0.001737 & 0.003448 & 7.99913 \\
\hline & G & 0.003782 & -0.001290 & -0.001861 & 7.99938 \\
\hline & B & -0.001807 & -0.000638 & 0.001907 & 7.99932 \\
\hline \multirow{3}{*}{$\begin{array}{l}\text { ref. } \\
{[16]}\end{array}$} & $\mathrm{R}$ & 0.0135 & \multirow{3}{*}{\multicolumn{2}{|c|}{ unknown }} & 7.99740 \\
\hline & $\mathrm{G}$ & -0.0835 & & & 7.99750 \\
\hline & B & -0.017 & & & 7.99690 \\
\hline
\end{tabular}

* mean for all colour planes 


\section{Conclusion}

Table 9. Values of NPCR, UACI and processing speeds

\begin{tabular}{lcccc}
\hline $\begin{array}{l}\text { reference } \\
\text { or image }\end{array}$ & $\begin{array}{c}\text { colour } \\
\text { plane }\end{array}$ & $\begin{array}{c}\text { NPCR } \\
(\%)\end{array}$ & $\begin{array}{c}\text { UACI } \\
(\%)\end{array}$ & $\begin{array}{c}v_{\text {proc }} \\
(\mathrm{MB} / \mathrm{s})\end{array}$ \\
\hline Lena 256-8 & - & 99.7984 & 33.4820 & 1.075115 \\
\hline ref. [15] & - & 99.6094 & 33.4635 & 1.953125 \\
\hline Lena & $\mathrm{R}$ & 99.8044 & 33.4744 & \\
256-24 & $\mathrm{G}$ & 99.7903 & 33.4818 & 1.072387 \\
& $\mathrm{~B}$ & 99.7865 & 33.4642 & \\
\hline ref. & $\mathrm{R}$ & 99.4048 & 33.2243 & \\
{$[17]$} & $\mathrm{G}$ & 99.3306 & 33.3345 & 0.451431 \\
& $\mathrm{~B}$ & 99.4845 & 33.2893 & \\
Lena & $\mathrm{R}$ & 99.8058 & 33.4621 & \\
$512-24$ & $\mathrm{G}$ & 99.8169 & 33.4477 & 1.056691 \\
& $\mathrm{~B}$ & 99.8168 & 33.4810 & \\
\hline ref. & $\mathrm{R}$ & 99.63 & 33.31 & \\
{$[16]$} & $\mathrm{G}$ & 99.60 & 33.34 & 3.083882 \\
& $\mathrm{~B}$ & 99.61 & 33.43 & \\
\hline
\end{tabular}

For comparing results of our proposal, we encrypted different versions of plaintext image lena utilized by other authors. Grayscale version with resolution of $256 \times 256$ pixels used in [15] is denoted in this subparagraphr as lena256-8. True colour image with the same resolution was used in [17] and here it is called lena256-24. Paper [16] used true colour version with resolution of $512 \times 512$ pixels, which is marked here as lena512-24. All encryptions done by our algorithm utilized key $K_{1}$.

Correlation coefficients $\rho$ and values of entropy $H$ for algorithms described in [15-17] and for the proposed approach are included in Tab. 8. The correlation coefficients were calculated separately for each colour plane $(R$ stands for red, $G$ for green and $B$ for blue colour plane) of encrypted images.

The results show that our solution obtains correlation coefficients comparable with those yielded by $[15,16]$. In some cases they are slightly better (colour planes of lena256-24 vs those of [17]). Values of entropy are better when compared with [17], but otherwise they are slightly worse.

Values of NPCR, UACI and computed processing speeds of encryption $v_{\text {proc }}$ are shown in Tab. 9. While NPCR and UACI are calculated separately for each colour plane, processing speed measures encryption of all three colour planes.

It could be concluded that our solution reaches values of NPCR and UACI which are comparable to those achieved by proposal described in [15]. Comparison with the other two approaches shows that our solution is better by means of NPCR and UACI values. On the other hand, our proposal is the second slowest of mentioned algorithms. Approach from [16] is three times faster, but the solution described in [17] is still more than two times slower than our proposal.
This paper proposed chaotic image encryption algorithm which utilizes skipping of iterations for providing higher robustness against phase space reconstruction attack. As the iterate skipping depends solely on key chosen by user, the details of this process are clear only when key is revealed.

Described encryption algorithm could be used in various applications which require secure transmission of image data. Some examples of those include sending pictures over internet, safe storage of image data, or providing higher amount of security in steganographic systems which use rather simple data hiding techniques [23, 24].

Properties of our proposal such as robustness against statistical or differential attacks were verified by experiments with set of four images and four keys. The set of images contained true colour and grayscale images with square and not square resolutions. Randomness of generated encrypted images was evaluated by tests from NIST 800-22 suite. Because all resulting $P$-values were higher than required significance level and also all proportions reached desired values, the tests were succesfully passed. Comparison with results achieved by other researchers shows that our algorithm reaches similar or even slightly better values of correlation coefficients and entropy, while the values of NPCR and UACI are usually clearly better. These advantages are balanced by slower processing speed, although one of the other approaches is even slower.

The processing speed as the biggest drawback of our proposal could be improved in future. However, as the described approach would always require more generated iterations than other algorithms, it may not reach the values achieved by the fastest designs. Still, there are some available options which would help to increase the processing speed, such as more effective usage of computer memory or enabling higher performance of computations. First approach merges some algorithm steps, eg generated iterations do not need to be stored in memory before their quantization, instead they could be passed to next iteration and could be stored in memory after the quantization. Second way is much more uncertain, because the iterations with more decimal places could be split into more values, but the higher precision usually causes increased computational complexity. Therefore careful considerations need to be made for improving processing speed.

\section{Acknowledgements}

This work was supported by following research grants: KEGA 023TUKE-4/2017 and VEGA 1/0772/17. 


\section{REFERENCES}

[1] ST Special Publication 800-38A: Recommendation for Block Cipher Modes of Operation: Methods and Techniques. 200166 p.[Online] Cited 2017-10-19.Available at: http://nvlpubs.nist.gov /nistpubs/Legacy/SP/nistspecialpublication800-38a.pdf.

[2] R. Matthews, "On the Derivation of a 'Chaotic' Encryption Algorithm", Cryptologia, 1989, vol. 8, no. 6, pp. 29-41.ISSN: 0161-1194.DOI: 10.1080/0161-118991863745.

[3] J. Fridrich, "Symmetric Ciphers based on Two-Dimensional Chaotic Maps", International Journal of Bifurcation and Chaos, 1998, vol. 8, no. 6, pp. 1259-1284.ISSN: 0218-1274. DOI: $10.1142 / \mathrm{S} 021812749800098 \mathrm{X}$.

[4] V. I. Arnold and A. Avez, Ergodic Problems of Classical Mechanics, New Jersey: W.A.Benjamin, 1968.

[5] B. Chirikov, Research Concerning the Theory of Non-Linear Resonance and Stochasticity, Geneva: CERN, 1971.

[6] R. May, "Simple Mathematical Models with Very Complicated Dynamics", Nature, 1976, vol. 261, no. 5560, pp. 459-467.ISSN: 0028-0836.DOI: $10.1038 / 261459 \mathrm{a} 0$.

[7] S. Li, G. Chen and X. Mou, "On the Dynamical Degradation of Digital Piecewise Linear Chaotic Maps", International Journal of Bifurcation and Chaos, 2005, vol. 15, no. 10, pp. 3119-3151. ISSN: 0218-1274. DOI: 10.1142/S0218127405014052.

[8] F. Takens, "On the Numerical Determination of the Dimension of an Attractor, Dynamical Systems and Bifurcations", Lecture Notes Mathematics vol. 1125, B. L. J.Braaksma, H. W. Broer, F. Takens (Eds), Berlin, Heidelberg: Springer, 1985, pp. 99-106. ISBN: 978-3-540-15233-0.DOI: 10.1007/BFb0075637.

[9] T. Sauer, J. A. Yorke and M. Casdagli, "Embedology", Journal of Statistical Physics, 1991, vol. 65, no. 3-4, pp. 579-616. ISSN: 0022-4715.DOI: 10.1007/BF01053745.

[10] B. Kliková and A. Raidl, "Reconstruction of Phase Space of Dynamical Systems using Method of time Delay", Proceedings of WDS'11. Praha (Czech Republic), 2011, pp. 83-87.ISBN: 978-8-073-78186-6.

[11] N. K. Pareek, V. Patidar and K. K. Sud, "Cryptography using Multiple One-Dimensional Chaotic Maps", Communications Nonlinear Science and Numerical Simulation, 2005, vol. 10, no. 7, pp. 715-723. ISSN: 1007-5704.DOI: 10.1016/j.cnsns.2004.03.006.

[12] H. Liu and X. Wang, "Colour Image Encryption based on One-Time Keys and Robust Chaotic Maps", Computers \& Mathematics with Applications, 2010, vol. 59, no. 10, pp. 33203327. ISSN: 0898-1221.DOI: 10.1016/j.camwa.2010.03.017.

[13] X. Wang, L. Teng and X. Qin, "A Novel Colour Image Encryption Algorithm based on Chaos", Signal Processing, 2012, vol. 92, no. 4, pp. 1101-1108. ISSN: 0165-1684. DOI: 10.1016/j. sigpro.2011.10.023.

[14] X. Wang and K. Guo, "A New Image Alternate Encryption Algorithm based on Chaotic Map", Nonlinear Dynamics, 2014, vol. 76, no. 4, pp. 1943-1950. ISSN: 0924-090X.DOI: 10.1007/s11071-014-1259-7.

[15] C. Zhu, "A Novel Image Encryption Scheme based on Improved Hyper-Chaotic Sequences", Optics Communications, 2012, vol. 285, no. 1, pp. 29-37.ISSN: 0030-4018. DOI: $10.1016 /$ j. optcom.2011.08.079.

[16] M. A. Murillo-Escobar, C. Cruz-Hernández, F. Abundiz-Pérez et al, "A RGB Image Encryption Algorithm based on Total Plain Image Characteristics and Chaos", Signal Processing, 2015, vol. 109, no. C, pp. 119-131. ISSN: 0165-1684. DOI: 10.1016/j.sigpro.2014.10.033.
[17] X. J. Tong, Z. Wang, M. Zhang et al, "An Image Encryption Algorithm based on the Perturbed High-Dimensional Chaotic Map", Nonlinear Dynamics, 2015, vol. 80, no. 3, pp. 1493-1508. ISSN: 0924-090X. DOI: 10.1007/s11071-015-1957-9.

18 C. E. Shannon, "A Mathematical Theory of Communication", The Bell System Technical Journal, 1948, vol. 27, no. 3, pp. 379423. ISSN: 0005-8580. DOI: 10.1002/j.1538-7305.1948.tb01338.x.

19] Y. Wu, J. Noonan and S. Agaian, "NPCR and UACI Randomness Tests for Image Encryption", Journal of Selected Areas Telecommunications, 2011, vol. 2, no. 4, pp. 31-38. ISSN: 1925-2676

[20] ST Internal Report 6483: Randomness Testing of the Advanced Encryption Standard Finalist Candidates, 200015 p. [Online] Cited 2017-11-02. Available at: http://ws680.nist.gov/publication/get_pdf.cf?pub_id/alpha 151216

[21] ST Special Publication 800-22 Rev.1a: A Statistical Test Suite for Random and Pseudorandom Number Generators for Cryptographic Applications. 2001131 p. [Online] Cited 2017-11-02. Available at: http:http://nvlpubs.nist.gov/nistpubs/Legacy/SP/ nistspecialpublication800-22r1a.pdf.

[22] High Resolution Images used for an Experiment with NIST 800-22 Suite, [Online] Cited 2017-11-13. Available at: http://imagedb.wz.sk/ sets/flowerandtarn.zip.

[23] J. Oravec and J. Turán, "Substitution Steganography with Security Improved by Chaotic Image Encryption", Proceedings of Informatics 2017. Poprad (Slovakia), pp. 284-288. ISBN: 978-1-538-60888-3 DOI: 10.1109/Informatics.2017.8327261.

[24] V. Hajduk, M. Broda, O. Kováč and D. Levický, "Image Steganography with QR Code and Cryptography", Proceedings of Radioelektronika 2016. Košice (Slovakia), pp. 350-353. ISBN: 978-15-0901-673-0. DOI: 10.1109/RADIOELEK.2016.7477370.

Received 22 November 2017

Jakub Oravec received his Ing (MSc) degree from Department of Electronics and Multimedia Communications (DEMC), Technical University of Košice in 2015. Now he continues as $\mathrm{PhD}$ student. His research interests include image encryption, steganography and digital image processing.

Ján Turán received Ing (MSc) degree in physical engineering with honours from the Czech Technical University, Prague, Czech Republic, in 1974, and RNDr degree in experimental physics with honours from Charles University, Prague, Czech Republic, in 1980. He received a CSc (PhD) and DrSc (DSc) degrees in radioelectronics from Technical University of Košice, Slovakia, in 1983, and 1992, respectively. Since March 1979, he has been at the Technical University of Košice as Full Professor for electronics and information technology. His research interests include digital signal processing and fiber optics, communication and sensing.

L’uboš Ovseník received his Ing (MSc) and $\mathrm{PhD}$ degrees from DEMC, Technical University of Košice in 1990 and 2002. He works at the Technical University of Košice, currently as an Associate Professor. His research interests include photonics, fiber optic communication systems and sensor networks.

Tomáš Huszaník received Ing (MSc) degree in 2017 at DEMC, Technical University of Košice. Since September 2017 he continues as $\mathrm{PhD}$ student. His research interests include all optical networks and degradation mechanisms in all optical WDM systems. 